\title{
Oral contraceptive pills and inherited thrombophilia in a young woman with deep venous thrombosis
}

R.A.R. Mahfouz, ${ }^{1}$ Z.K. Otrock, ${ }^{2}$ M.A. Ghasham, ${ }^{1}$ A.S. Sabbagh, ${ }^{1}$ A. T. Taher ${ }^{2}$ and A. Bazarbachi ${ }^{2}$

\section{Introduction}

Inherited risk factors for vascular disease include factor $\mathrm{V}$ Leiden [1], prothrombin [2] and methylenetetrahydrofolate reductase (MTHFR) point mutations [3]. The use of oral contraceptives is also a recognized risk factor for venous thromboembolism [4]. In Eastern Mediterranean countries, the highest frequency of factor $\mathrm{V}$ Leiden was reported in Lebanon (14\%) [5,6]. However, there are no studies related to awareness of the use of oral contraceptive pills among individuals with a high risk for thrombotic events.

Here we present the case of a female patient on oral contraceptive pills who sustained an extensive deep venous thrombosis (DVT). The patient was found to be heterozygous for factor V Leiden (G1691A) and homozygous for the MTHFR (C677T) mutations.

\section{Case report}

Our patient, a 21-year-old Lebanese woman, had been hospitalized in another hospital 5 months previously for pain and swell- ing of the left lower extremity. She also complained of low back pain of the same duration. She was a non-smoker with no known medical problems. Her body mass index was $22 \mathrm{~kg} / \mathrm{m}^{2}$. She was taking combined oral contraceptive pills for her irregular menstrual periods. Venous doppler ultrasonography showed extensive DVT involving the entire length of the common femoral, popliteal and calf veins extending into the left external iliac and common iliac veins. She had initially received anticoagulant treatment with heparin and was later discharged on warfarin $5 \mathrm{mg}$ /day to maintain an international normalized ratio of 2.5-3.0. The patient had no family history of thrombotic events.

The patient was seen in our institution for follow-up. In addition to the regular blood and chemistry studies, she was screened for acquired and inherited thrombophilia. The work-up revealed that the patient was heterozygous for factor V Leiden (G1691A) and homozygous for MTHFR (C677T) mutations (Figure 1) with a normal serum homocysteine level. The work-up also included fibrinogen level, factors VII and VIII activities, lupus anticoagulant and

${ }^{1}$ Department of Pathology and Laboratory Medicine; ${ }^{2}$ Department of Internal Medicine, American University of Beirut Medical Centre, Beirut, Lebanon (Correspondence to R.A.R. Mahfouz: rm11@aub.edu.lb).

Received: 06/04/06; accepted: 30/07/06

المجلة الصحية لشرق المتوسط، منظمة الصحة العالمية، المجلد الخامس عشر، العدد (، 9 +. 

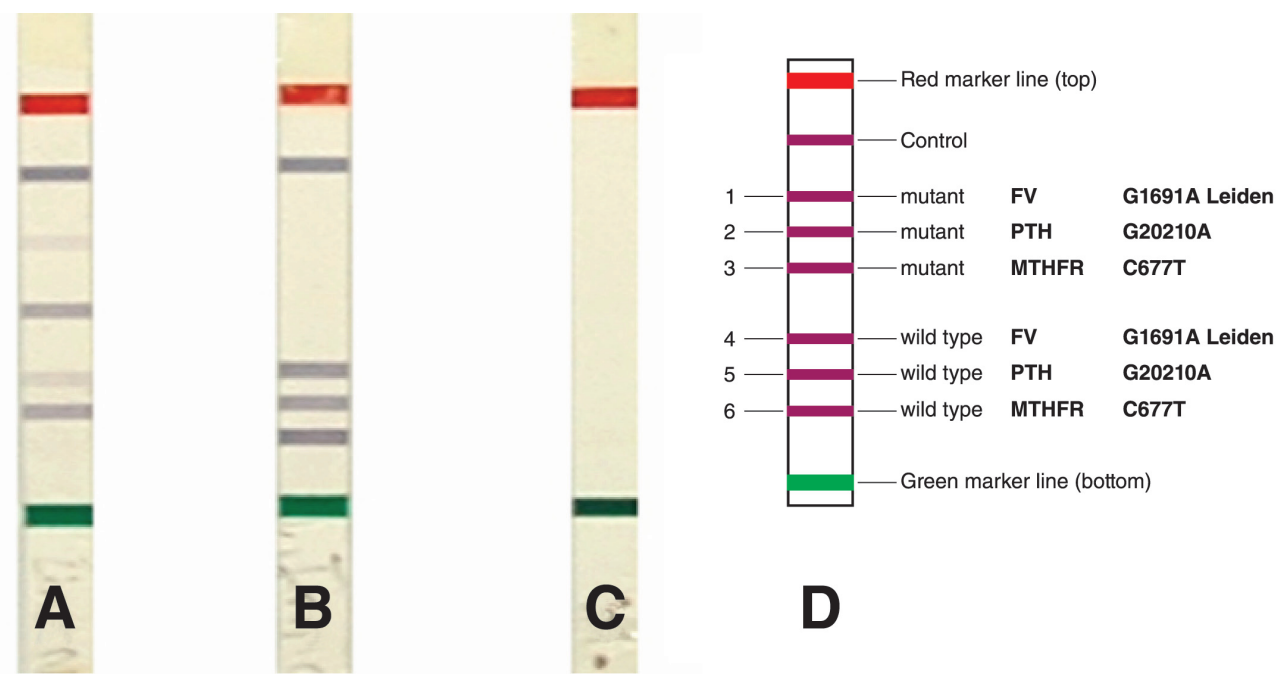

Figure 1 Staining pattern of hybridized DNA from patient (A), normal positive control (B), negative control (C) and Viennalab Stripassay (D). Insert shows pattern of hybridization

anticardiolipin/antiphospholipid antibodies, antithrombin III, protein $\mathrm{S}$ and protein $\mathrm{C}$. DNA was extracted from the sample according to the extraction protocol supplied by the manufacturer (factor V StripAssay, ViennaLab, Austria). Briefly, a $100 \mu \mathrm{L}$ blood sample was lysed twice in red blood cell lysis buffer. The reaction tubes were run at the following conditions: $940 \mathrm{C}$ for 2 min followed by 30 cycles of $940 \mathrm{C}$ for $15 \mathrm{~s}, 580 \mathrm{C}$ for $30 \mathrm{~s}$, and $720 \mathrm{C}$ for $30 \mathrm{~s}$. Hybridization of the amplified product to the test strips was then conducted, followed by 2 stringent washing steps and colour development. The samples were interpreted by comparing the staining pattern of the corresponding processed test strip with the decoder table provided by the manufacturer. The targeted mutations of the above assay are: factor V G1691A, factor II G20210A and MTHFR C677T.

The patient's activated protein $C$ resistance was 1.8 (reference range $>2.1$ ). She had an identical twin sister who had the same thrombophilia profile with no associated events. The patient's father was found to be homozygous for factor $\mathrm{V}$ Leiden and heterozygous for the MTHFR mutations, while her mother was normal for factor $\mathrm{V}$ and heterozygous for MTHFR gene mutations. The patient was advised not to use oral contraceptives. She continued her oral anticoagulation for 9 months, and the follow-up venous doppler ultrasonography showed recanalization of the thrombosed veins. Two years after the incident, the patient is not on oral contraceptive pills and is doing well with no recurrence of DVT.

\section{Discussion}

Factor V Leiden is the most common inherited risk factor for venous thrombosis. The risks are estimated to be increased up to $5-10$-fold for heterozygous and $50-100$ fold for homozygous adults respectively [7]. In Eastern Mediterranean countries, a 
high prevalence of factor $\mathrm{V}$ Leiden has been reported in healthy individuals, ranging from $7 \%$ to $14 \%$, with the highest frequency reported in Lebanon $(14 \%)[5,6]$.

The prevalence of MTHFR C677T mutation in Lebanon has been assessed. It was found that Lebanon has a relatively high prevalence of the homozygous $\mathrm{T} / \mathrm{T}$ genotype $(11.04 \%)$ compared to that seen in European countries, including Germany $(10.4 \%)$ and Greece $(10.0 \%)$, and southeast Asian communities [8].

The use of combined oral contraceptives is a recognized risk factor for venous thromboembolism, and their use is itself associated with a 2-4-fold increase in the risk of venous thromboembolism, regardless of the presence of other genetic or acquired risk factors [4]. Progestin-only contraceptives were shown to be a safer alternative to the combined oral contraceptive pills [9]. The presence of inherited thrombophilia increases the risk of venous thromboembolism due to oral contraceptives up to an absolute risk of 3/1000 person-years, in comparison with the baseline risk of 3-6/10 000 person-years [10]. It is well known that oral contraceptive treatment often constitutes a trigger factor for venous thromboembolism, very often the first episode, when given to women who carry an unrecognized thrombophilic condition [11]. The question has been raised whether it is worth screening women for thrombophilic alterations before the use of oral contraceptives [12,13].

It has been previously reported that factor $\mathrm{V}$ mutations display a strong interaction with oral contraceptive use [14], with heterozygous carriers using oral contraceptives having a $30-50$-fold increased risk of thrombosis compared with women who do not use oral contraceptives and who have a normal factor $\mathrm{V}$ genotype.
Our patient was on oral contraceptives and developed DVT. She was found to be heterozygous for factor V Leiden and homozygous for the MTHFR mutations, which increased her risk of thrombosis. She had a twin sister with the same thrombophilia profile but no associated thrombotic events. In their interesting article, Salomon et al. determined the prevalence of single and combined prothrombotic factors in patients with idiopathic venous thromboembolism and estimated the associated risks in a study group consisting of 162 patients referred for work-up of thrombophilia [15]. Two or more polymorphisms were detected in 27 patients $(16.7 \%)$ and in 3 of 336 controls $(0.9 \%)$. Logistic regression analysis disclosed odds ratios of 58.6 for joint occurrence of factor $\mathrm{V}$ and factor II polymorphisms, of 35.0 for factor $\mathrm{V}$ and MTHFR polymorphisms, and of 7.7 for factor II and MTHFR polymorphisms. The authors concluded that the presence of more than one of the prothrombotic polymorphisms was associated with a substantial risk of venous thromboembolism.

Whether patients in our region should be screened for inherited thrombophilia before taking oral contraceptives is not known, albeit we recognize the relatively high prevalence of these mutations in the Eastern Mediterranean Region. It is also important to state that cost-benefit analysis studies should be conducted to evaluate whether screening for factor $\mathrm{V}$, factor II, and MTHFR gene mutations (expensive testing) is cost-effective or not regarding morbidity or mortality incurred by oral contraceptive pills users. This has not been previously investigated in a high-prevalence setting such as in Lebanon (and the Eastern Mediterranean Region in general). We open the door for future research in this field. 


\section{References}

1. Dahlback B. Resistance to activated protein $C$ caused by the factor VR506Q mutation is a common risk factor for venous thrombosis. Thrombosis and haemostasis, 1997, 78(1):483-8.

2. Poort SR et al. A common genetic variation in the 30-untranslated region of the prothrombin gene is associated with elevated plasma prothrombin levels and an increase in venous thrombosis. Blood, 1996, 88(10):3698-703.

3. Graham IM et al. Plasma homocysteine as a risk factor for vascular disease. The European Concerted Action Project. Journal of the American Medical Association, 1997, 277(22):1775-81.

4. Helmerhorst FM et al. Oral contraceptives and thrombotic disease: risk of venous thromboembolism. Thrombosis and haemostasis, 1997, 78(1):327-33.

5. Irani-Hakime $\mathrm{N}$ et al. The prevalence of factor V R506Q mutation-Leiden among apparently healthy Lebanese. American journal of hematology, 2000, 65(1):45-9.

6. Taher A et al. High prevalence of Factor $\mathrm{V}$ Leiden mutation among healthy individuals and patients with deep venous thrombosis in Lebanon: is the Eastern Mediterranean region the area of origin of this mutation? Thrombosis and haemostasis, 2001, 86(2):723-4.

7. Dahlbäck B. Inherited thrombophilia: resistance to activated protein $\mathrm{C}$ as a pathogenic factor of venous thromboembolism. Blood, 1995, 85:607-14.

8. Almawi WY et al. Differences in the frequency of the C677T mutation in the methylenetetrahydrofolate reductase
(MTHFR) gene among the Lebanese population. American journal of hematology, 2004, 76(1):85-7.

9. Gomes MP, Deitcher SR. Risk of venous thromboembolic disease associated with hormonal contraceptives and hormone replacement therapy: a clinical review. Archives of internal medicine, 2004, 164(18):1965-76.

10. De Stefano $V$ et al. Inherited thrombophilia, pregnancy, and oral contraceptive use: clinical implications. Seminars in vascular medicine, 2003, 3(1):47-60.

11. Pabinger I et al. The risk of thromboembolism in asymptomatic patients with protein $\mathrm{C}$ and protein $\mathrm{S}$ deficiency: a prospective cohort study. Thrombosis and haemostasis, 1994, 1994, 71(4):441-5.

12. Hellgren $\mathrm{M}$ et al. Resistance to activated protein $\mathrm{C}$ as a basis for venous thromboembolism associated with pregnancy and oral contraceptives. American journal of obstetrics and gynecology, 1995, 173(1):210-3.

13. Vandenbroucke JP et al. Factor V Leiden: should we screen oral contraceptive users and pregnant women? British medical journal, 1996, 313:1127-30.

14. Vandenbroucke JP et al. Increased risk of venous thrombosis in oral-contraceptive users who are carriers of factor $\mathrm{V}$ Leiden mutation. Lancet, 1994, 344:1453-7.

15. Salomon $O$ et al. Single and combined prothrombotic factors in patients with idiopathic venous thromboembolism: prevalence and risk assessment. Arteriosclerosis, thrombosis, and vascular biology, 1999, 19(3):511-8. 